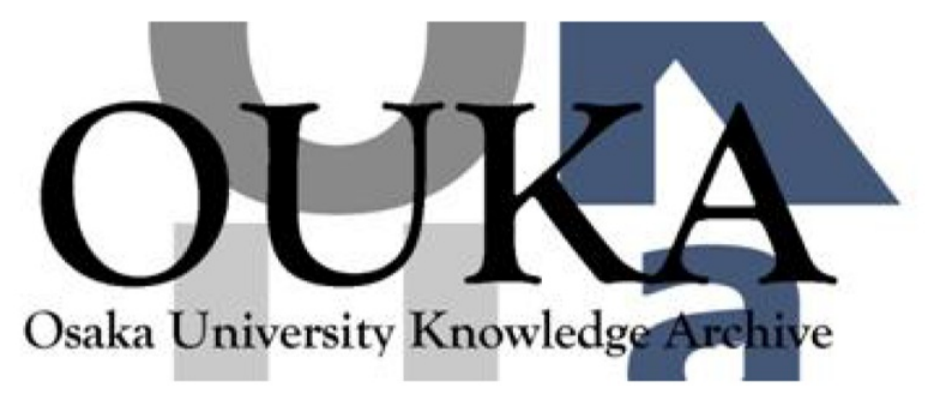

\begin{tabular}{|c|l|}
\hline Title & $\begin{array}{l}\text { A low-power technique for pipelined ADCs with } \\
\text { programmable gain amplification }\end{array}$ \\
\hline Author(s) & $\begin{array}{l}\text { Okura, Tetsuro; Okura, Shunsuke; Matsuoka, } \\
\text { Toshimasa et al. }\end{array}$ \\
\hline Citation & IEICE Electronics Express. 10(1) p. 20120876 \\
\hline Issue Date & $2013-01$ \\
\hline oaire:version & VoR \\
\hline URL & https://hdl. handle. net/11094/51662 \\
\hline rights & copyright $\odot 2013$ IEICE \\
\hline Note & \\
\hline
\end{tabular}

Osaka University Knowledge Archive : OUKA

https://ir. Library. osaka-u. ac. jp/

Osaka University 


\title{
A low-power technique for pipelined ADCs with progr- ammable gain amplification
}

\author{
Tetsuro Okura ${ }^{a)}$, Shunsuke Okura, Toshimasa Matsuoka, \\ and Kenji Taniguchi \\ Division of Electrical, Electronic and Information Engineering, Osaka University, \\ 2-1 Yamada-oka, Suita, Osaka, 565-0871 Japan \\ a) ohkura@si.eei.eng.osaka-u.ac.jp
}

Abstract: A pipelined analog-to-digital converter (ADC) has been investigated, which has a programmable gain achieved by the gain control in a first-stage multiplying digital-to-analog converter (MDAC). The current consumption reduction under low gain is realized by controlling the transconductance and compensation capacitor of the MDAC circuit according to the input gain. The pipelined ADC designed using a $0.18 \mu \mathrm{m}$ CMOS technology shows a sampling rate of $40 \mathrm{MSps}$ and an input gain of $0-18 \mathrm{~dB}$ ( $6 \mathrm{~dB}$-step). The maximum current consumption is $14.2 \mathrm{~mA}$ at the input gain of $18 \mathrm{~dB}$ and the minimum is $7.5 \mathrm{~mA}$ at $0 \mathrm{~dB}$. The signal-to-noise plus distortion ratio (SNDR) is $66.1 \mathrm{~dB}$ for an input signal amplitude of $2 \mathrm{Vpp}$ and an input gain of $0 \mathrm{~dB}$, and $63.4 \mathrm{~dB}$ for an input signal amplitude of $250 \mathrm{mVpp}$ and an input gain of $18 \mathrm{~dB}$.

Keywords: pipelined A/D converter, programmable gain amplifier, controllable transconductance, low power

Classification: Integrated circuits

\section{References}

[1] S. Nitta and K. Tanaka, "A $79 \mathrm{~dB}-\mathrm{SNR} 70 \mathrm{~mW} 18 \mathrm{MHz}$ CCD front-end with fully-digital amplification scheme," IEEE Trans. Consum. Electron., vol. 47, no. 3, pp. 459-465, Aug. 2001.

[2] T. Iida, T. Akahori, M. A. B. Mustafa, K. Yasutomi, and S. Kawahito, "An A/D converter with a built-in variable gain amplifier for CMOS Image Sensors," ITE Technical Report, vol. 34, no. 29, pp. 85-88, July 2010.

[3] C. C. Hsu and J. T. Wu, "A Highly Linear 125-MHz CMOS SwitchedResistor Programmable-Gain Amplifier," IEEE J. Solid-State Circuits, vol. 38, no. 10, pp. 1663-1670, Oct. 2003.

[4] S. H. Lewis, H. S. Fetterman, G. F. Gross, Jr., R. Ramachandran, and T. R. Viswanathan, "A 10-b 20-Msample/s Analog-to-Digital Converter," IEEE J. Solid-State Circuits, vol. 27, no. 3, pp. 351-358, March 1992.

[5] T. Okura, S. Okura, T. Matsuoka, and K. Taniguchi, "A Low Power Technique for Programmable Gain Amplifier with Controllable input/output Transconductance and Compensation Capacitor," IEICE Trans. Electron., vol. J95-C, no. 7, pp. 131-138, July 2012. 


\section{Introduction}

Pipelined ADCs are widely used as the analog front-end in systems requiring resolutions over 10-bits and sampling rates of several tens of MSps, such as communications equipment and image processing systems. A programmable gain amplifiers (PGAs) are often required as front stage of ADC in order to effectively exploit ADC resolution even with low input signal amplitudes. However, the power consumption of PGAs must be very large to drive the sampling capacitor of the ADC. A method has been proposed to eliminate the need for a PGA in the analog region, which enlarges the resolution of the ADC itself and instead uses a PGA in the digital region, thereby reducing the number of op-amps used in the analog front-end and lowering the power consumption [1]. However, when the input signal amplitude is sufficiently large and the PGA operates at low gain, the ADC resolution goes above a requirement because a required resolution becomes lower. Thus the power efficiency at low gain mode becomes lower. Another method has also been proposed that introduces programmable gain by switching between the capacitors in a cyclic ADC, thereby reducing the number of op-amps [2]. However, since the programmable gain and analog-to-digital conversion would operate in sequence, this method cannot be adapted to pipelined ADCs having rapid throughput. Furthermore, since the feedback factor is controlled in the programmable gain operation, the bandwidth and phase margin change according to the gain setting, which degrades the power efficiency during low gain operation [3].

In this letter, we propose a method for obviating PGA while maintaining the high throughput of the pipelined ADC, by embedding a programmable gain amplification in the first-stage MDAC of a pipelined ADC, and executing the programmable gain amplification simultaneously with A/D conversion. The power consumption at lower gain operation is reduced by controlling the transconductance of the input and output stages and the phase compensation capacitor of 2-stage op-amp used in the first-stage MDAC according to input gain.

\section{Proposed circuit}

Figure 1 (a) shows a block diagram of the proposed pipelined ADC with programmable gain amplification. The first stage consists of a 1.5 bit flash ADC and a 1.5 bit MDAC with an input gain of $6-24 \mathrm{~dB}$ ( $6 \mathrm{~dB}$ step). Generally, the input gain of a 1.5 bit MDAC is $6 \mathrm{~dB}$, but providing a controllable range of $6-24 \mathrm{~dB}$ allows the pipelined ADC input gain to be controlled between 0 to $18 \mathrm{~dB}$. Considering the power consumption of the flash ADC and the maximum value for the MDAC gain, an accuracy of first stage MDAC is equivalent to 4.5 bit when the input gain is $18 \mathrm{~dB}$ (the MDAC gain is $24 \mathrm{~dB}$ ).

The second and third stages consist of 3.5 bit MDAC circuits with 3.5 bit flash ADCs and a gain of $18 \mathrm{~dB}$. The final stage employs a 3.5 bit flash ADC, resulting in a pipelined $\mathrm{ADC}$ with an overall resolution of 11 bits and an input gain of $0-18 \mathrm{~dB}$ ( $6 \mathrm{~dB}$ step). 


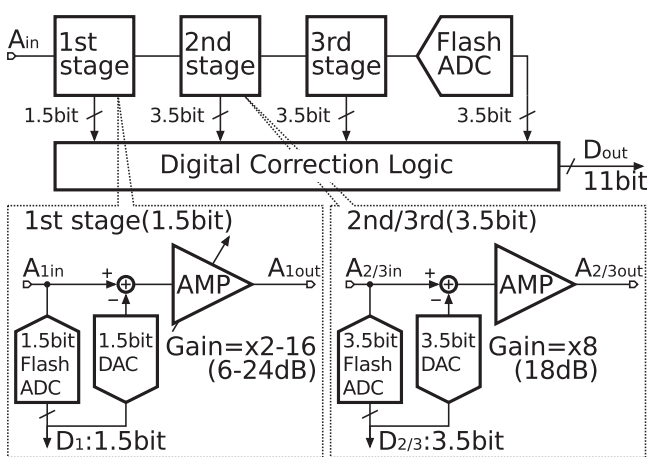

(a)

\begin{tabular}{|l|c|c|c|c|}
\hline Gain [dB] & 0 & 6 & 12 & 18 \\
\hline Vos,sys [V] & $7 / 32$ & $3 / 32$ & $1 / 32$ & 0 \\
\hline Vos,comp [mV] & \multicolumn{4}{|c|}{31.25} \\
\hline Total offset [mV] & 250 & 125 & 62.5 & 31.25 \\
\hline $\begin{array}{l}\text { Offset Correctable } \\
\text { range [mV] }\end{array}$ & 250 & 125 & 62.5 & 31.25 \\
\hline
\end{tabular}

(c)

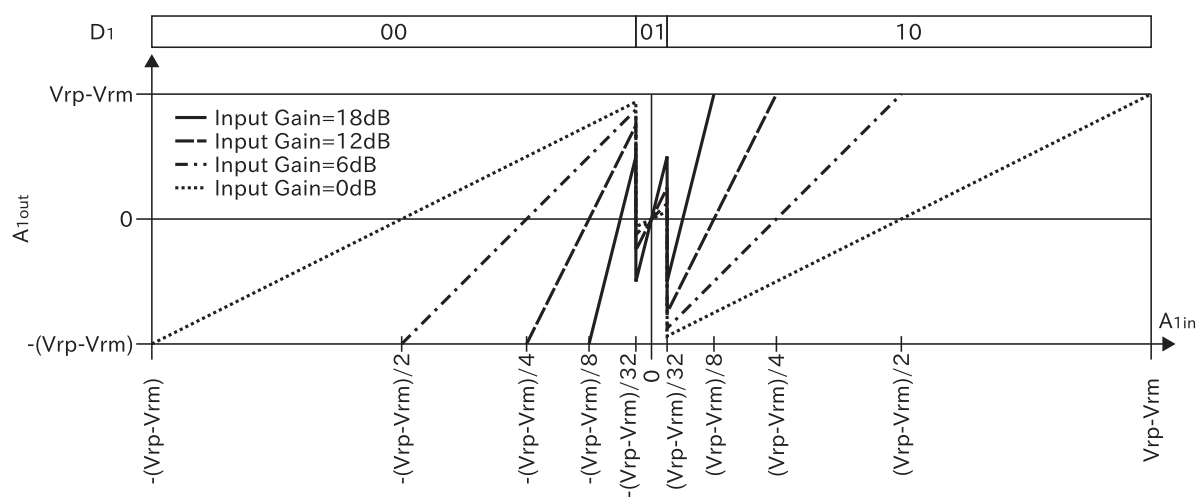

(b)

Fig. 1. (a) Block diagram of pipelined ADC with programmable gain amplification, (b) transfer function of the first pipelined stage, and (c) offset voltage of flash $\mathrm{ADC}$ at first stage $\left(V_{r p}-V_{r m}=1 V\right)$.

Figure 1 (b) shows the input-output transfer function of the first-stage MDAC for each input gain. A threshold voltage of $\pm\left(V_{r p}-V_{r m}\right) / 32$ is employed in the flash ADC regardless of the input gain. Since the threshold voltage of the flash ADC is not controlled depending on the input gain, additional floating switches are not needed. Generally, in a 1.5 bit pipelined stage with the maximum input signal amplitude of $\pm a\left(V_{r p}-V_{r m}\right)$, the threshold voltage for the flash ADC is set at $\pm a\left(V_{r p}-V_{r m}\right) / 4$. By keeping the input offset voltage for the comparator used in the flash $\mathrm{ADC}$ within $\pm a\left(V_{r p}-V_{r m}\right) / 4$, the influence of the comparator input offset voltage can be compensated for by a digital correction circuit [4]. In the proposed circuit, for input gains of $18,12,6$ and $0 \mathrm{~dB}$, the maximum input signal amplitudes are $\pm\left(V_{r p}-V_{r m}\right) / 8$, $\pm\left(V_{r p}-V_{r m}\right) / 4, \pm\left(V_{r p}-V_{r m}\right) / 2$ and $\pm\left(V_{r p}-V_{r m}\right)$, respectively. As the threshold voltage for the flash $\mathrm{ADC}$ is set to the identical value of $\pm\left(V_{r p}-V_{r m}\right) / 32$ for all input gain modes, the transfer functions for the 12, 6 and $0 \mathrm{~dB}$ modes are equivalent to the cases for the comparator with systematic input offsets $\left(V_{\text {os }, \text { sys }}\right)$ of $\left(V_{r p}-V_{r m}\right) / 32,3\left(V_{r p}-V_{r m}\right) / 32$ and $7\left(V_{r p}-V_{r m}\right) / 32$, respectively. As shown in Figure 1 (c), when the differential reference voltage $\left(V_{r p}-V_{r m}\right)$ is $1 \mathrm{~V}$, the systematic offset and the comparator offset is corrected with the digital correction circuit at all input gain by maintaining the comparator offset voltage $\left(V_{o s, c o m p}\right)$ below $31.25 \mathrm{mV}$. 

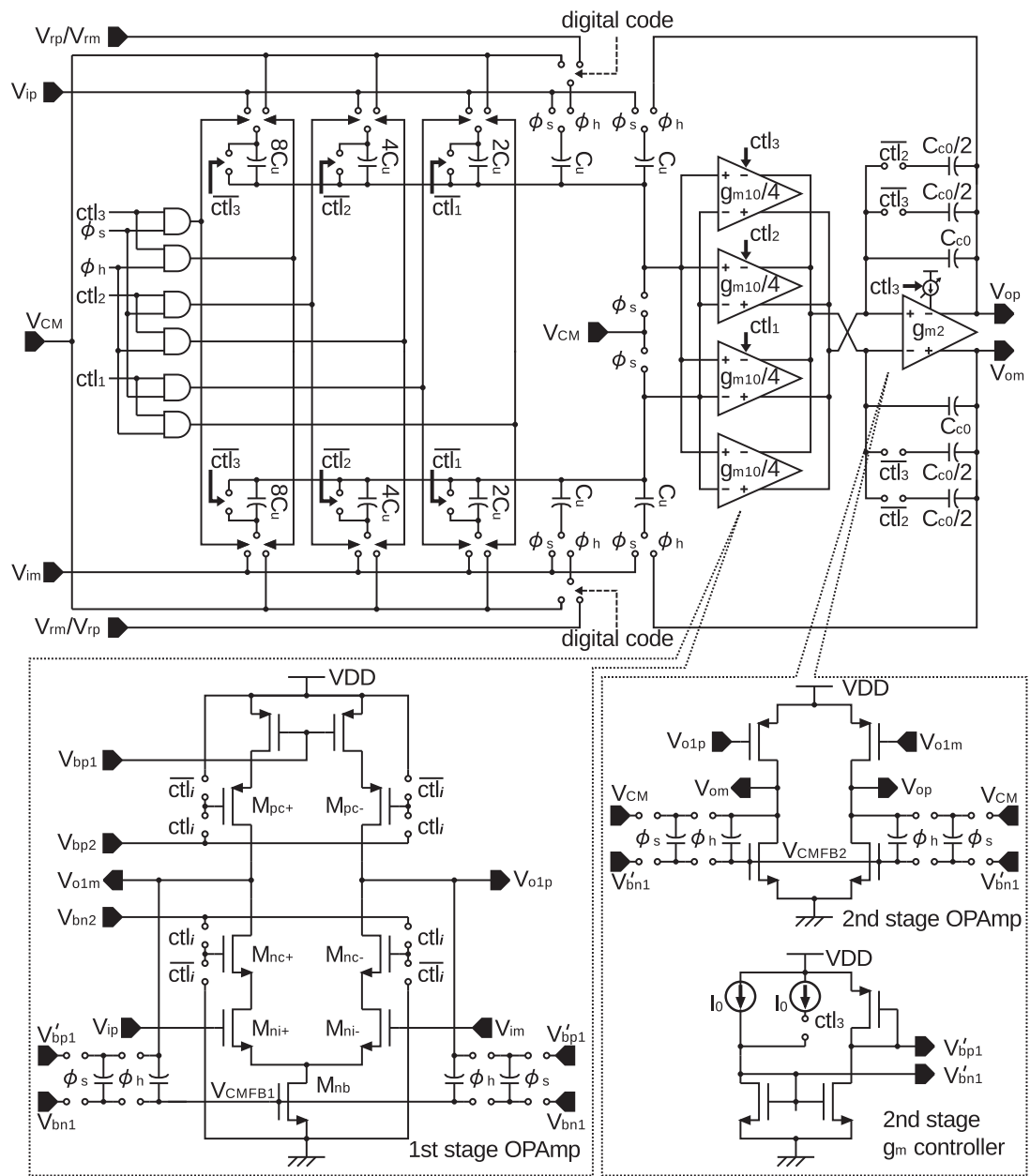

(a)

\begin{tabular}{|c|c|c|c|c|}
\hline Input gain[dB] & 0 & 6 & 12 & 18 \\
\hline$\beta_{\mathrm{F}}$ & $1 / 2$ & $1 / 4$ & $1 / 8$ & $1 / 16$ \\
\hline \hline $\mathrm{gm} 1$ & $1 / 4$ & $2 / 4$ & $3 / 4$ & 1 \\
\hline $\mathrm{gm} 2$ & 0.7 & 0.7 & 0.7 & 1 \\
\hline $\mathrm{Cc}$ & 2.0 & 2.0 & $3 / 2$ & 1 \\
\hline
\end{tabular}

(b)

Fig. 2. (a) MDAC circuit of the first stage with programmable gain and (b) its key parameters normalized by ones for input gain of $18 \mathrm{~dB}$.

Figure 2 (a) shows the MDAC with programmable gain amplification. Programmable gain is achieved by controlling the number of sampling capacitors. In $0 \mathrm{~dB}$ mode, $c t l_{i}(\mathrm{i}=1,2,3)$ digits are all set to "Low", the input signal is sampled by $2 C_{u}$, and the feedback capacitor during amplification is $C_{u}$, so the gain of the MDAC is two $(6 \mathrm{~dB})$. In $18 \mathrm{~dB}$ mode, all the $c t l_{i}$ digits are set to "High", the input signal is sampled by $16 C_{u}$, and the feedback capacitor during amplification is $C_{u}$. As a result, the gain of the MDAC circuit is $16(24 \mathrm{~dB})$. In the same way, $c t l_{1}=$ "High", $c t l_{2}=$ "Low", and $c t l_{3}=$ "Low" are set for $6 \mathrm{~dB}$ mode, and $c t l_{1}=$ "High", $c t l_{2}=$ "High", and $c t l_{3}=$ "Low" for $12 \mathrm{~dB}$ mode.

In a switched capacitor circuit with a programmable gain amplification, 
the bandwidth and phase margin changes depending on input gain, causing lower power efficiency $[3,5]$. Controlling the transconductance of the input and output stages and the compensation capacitor of the 2-stage op-amp appropriately according to the gain, enhances the power efficiency at low gain [5]. A reduction in power consumption at low input gains is obtained by applying this technique to the MDAC of the proposed pipelined ADC.

Bandwidth $\left(\omega_{c}\right)$ and phase margin $\left(\phi_{m}\right)$ of MDAC composed of 2-stage opamp with compensation capacitor are given by

$$
\begin{aligned}
& \omega_{c}=\omega_{2}\left[\sqrt{\frac{1}{4}+\left(\frac{\omega_{1}}{\omega_{2}}\right)^{2}}-\frac{1}{2}\right]^{\frac{1}{2}}, \phi_{m}=\tan ^{-1}\left[\sqrt{\frac{1}{4}+\left(\frac{\omega_{1}}{\omega_{2}}\right)^{2}}-\frac{1}{2}\right]^{-\frac{1}{2}}, \\
& \omega_{1}=\beta_{F} \frac{g_{m 1}}{C_{C}}, \quad \omega_{2}=\frac{g_{m 2}}{C_{L}+C_{p 2}+C_{L} C_{p 2} / C_{C}}
\end{aligned}
$$

where $\beta_{F}\left(=(2 G)^{-1}\right)$ is the feedback factor of MDAC, $G$ is the input gain of ADC, $g_{m 1}$ and $g_{m 2}$ are the transconductance of input and output stages respectively, $C_{C}, C_{L}$ and $C_{p 2}$ are the phase compensation capacitor, the load capacitor and the input parasitic capacitor of output stage respectively. The power consumption at low gain mode is reduced by suppressing the changes of $\omega_{c}$ and $\phi_{m}$ caused by change of $\beta_{F}$. Figure 2 (b) shows $g_{m 1}, g_{m 2}$, and $C_{C}$ for each input gain. The value of each parameter is normalized so that it is unity in the $18 \mathrm{~dB}$ input gain. The $g_{m 1}$ is controlled linearly against the $\beta_{F}$ controlled exponentially. $C_{C}$ is controlled to make the $\omega_{c}$ constant. The change of $\omega_{2}$ caused by the variation of $C_{C}$ is suppressed through controlling $g_{m 2}$. As a result, the power efficiency at low gain mode is improved without large increase in a noise and die-area [5]. In addition, the factor of 0.7 is determined considering $C_{p 2}$ and the load capacitance including the influence of the feedback capacitors to achieve over $100 \mathrm{MHz}$ bandwidth [5], and it will slightly vary depending on a target specification.

The op-amps in the MDAC consist of an input stage in which $c t l_{i}$ varies the number of operating parallel-connected amplifiers to control $g_{m 1}$, an output stage which has variable $g_{m 2}$ achieved by controlling the bias voltage, and a controllable $C_{C}$.

$g_{m 1}$ is controlled by the number of operating parallel-connected amplifiers, so the current consumption $I_{1}$ in the input stage is proportional to $g_{m 1} \cdot g_{m 2}$ is controlled by varying the bias voltage $V_{b n 1}^{\prime}$ and $V_{b p 1}^{\prime}$ in the output stage, so the consumption current $I_{2}$ in the output stage is proportional to the square of $g_{m 2}$. Assuming the ratio $I_{1} / I_{2}$ of $1 / 2$, the current consumption in the first-stage MDAC at the $0 \mathrm{~dB}$ mode is achieved to be reduced by about $60 \%$ from at $18 \mathrm{~dB}$ mode.

Even if the PGA is still embedded in the analog front-end to achieve the required $\mathrm{ADC}$ resolution, the proposed technique can reduce the required gain variation in the PGA. By the adequate design, power consumption reduction in the overall analog front-end is expected even considering the increase of the required drivability of PGA due to the variation of sampling capacitance of the ADC. 


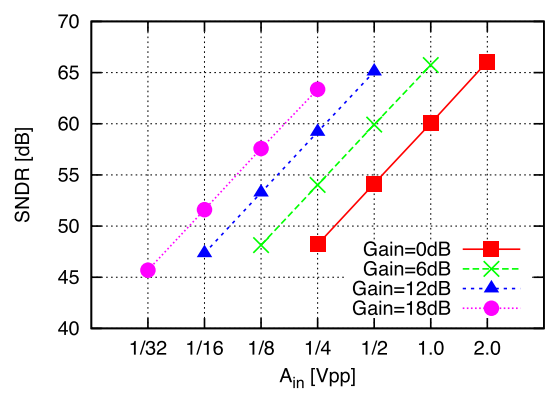

(a)

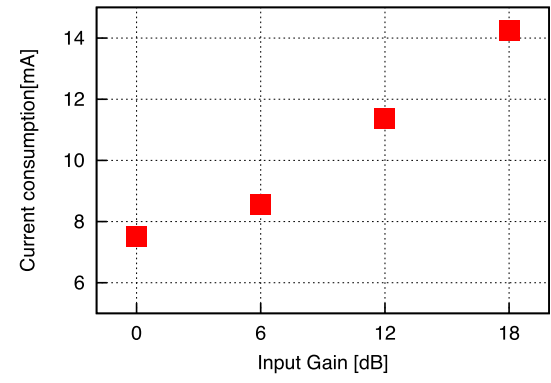

(b)

Fig. 3. Simulation results of the designed pipelined ADC. (a) Input voltage gain versus SNDR and (b) input voltage gain versus current consumption.

\section{Simulation results}

A pipelined $\mathrm{ADC}$ with a programmable input gain amplitude of $0-18 \mathrm{~dB}$ (6 dB step) and 11-bit resolution has been designed using a $0.18 \mu \mathrm{m}$ CMOS process with $3 \mathrm{~V}$ devices and metal-insulator-metal capacitors. The operating supply voltage is $3.3 \mathrm{~V}$. Figure 3 (a) shows simulation result of the relationship between the differential input signal amplitude (Ain) and the signal-to-noise plus distortion ratio (SNDR) at each input gain. The SNDR is calculated by applying an 8192-point FFT to the results of Spectre transient noise simulation with a throughput of $40 \mathrm{MSps}$ and an input signal frequency of $97.65625 \mathrm{kHz}$. Under an input signal amplitude of $250 \mathrm{mVpp}$, it is confirmed that increasing the gain of the first-stage MDAC improves the SNDR by about $6 \mathrm{~dB}$ per step. For an input signal amplitude of $2.0 \mathrm{Vpp}$ and an input gain of $0 \mathrm{~dB}$, the maximum SNDR was $66.1 \mathrm{~dB}$, and for an input signal amplitude of $250 \mathrm{mVpp}$ and an input gain of $18 \mathrm{~dB}$, the maximum SNDR was $63.4 \mathrm{~dB}$. Thus, a high SNDR is confirmed even at low signal amplitudes. The SNDR at maximum amplitude is degraded as the input gain increased because the output referred $\mathrm{kT} / \mathrm{C}$ noise in the first-stage MDAC increases in proportion to the square root of the input gain.

Figure 3 (b) shows the mean current consumption during operation under each gain mode. The highest current consumption is $14.2 \mathrm{~mA}$, occurring under an input gain of $18 \mathrm{~dB}$. It is lowest in the $0 \mathrm{~dB}$ mode, at $7.5 \mathrm{~mA}$. Thus, power consumption reduction of $47 \%$ is confirmed compared to the $18 \mathrm{~dB}$ case.

\section{Conclusion}

This letter has discussed low power technique for a pipelined ADC with programmable gain amplification. Programmable gains of $0-18 \mathrm{~dB}$ (6 dB step) are successfully implemented by controlling the number of sampling capacitors in the first-stage MDAC circuit. A simulation result confirms a SNDR value of $66.1 \mathrm{~dB}$ for an input signal amplitude of $2.0 \mathrm{Vpp}$ and an input gain of $0 \mathrm{~dB}$, and $63.4 \mathrm{~dB}$ for an input signal amplitude of $250 \mathrm{mVpp}$ and an input gain of $18 \mathrm{~dB}$. Controlling the transconductance of the input and output 
stages and the phase compensation capacitance of the 2-stage op-amp in the first-stage MDAC circuit according to the gain provides a power consumption reduction at low gain and leads to a high power efficiency. In a design using a $0.18 \mu \mathrm{m}$ CMOS process, the maximum current consumption is $14.2 \mathrm{~mA}$ for an input gain of $18 \mathrm{~dB}$ and the lowest is $7.5 \mathrm{~mA}$ for an input gain of $0 \mathrm{~dB}$. A $47 \%$ improvement of the power consumption at the input gain of $0 \mathrm{~dB}$ is achieved by using the proposed circuit techniques.

\section{Acknowledgments}

The present study was supported by the Ministry of Education, Culture, Sports, Science and Technology of Japan through a grant to the Osaka University Global COE Program, "Center for Electronic Devises Innovation". The VLSI chip in the present study was designed as part of the chip fabrication program of the VLSI Design and Education Center (VDEC) at the University of Tokyo in collaboration with Cadence Design Systems, Inc. 